\title{
Skull Base Osteomyelitis: A Comprehensive Imaging Review
}



\begin{abstract}
SUMMARY: Skull base osteomyelitis is a relatively rare condition, generally occurring as a complication of advanced otologic or sinus infection in immunocompromised patients. Skull base osteomyelitis is generally divided into 2 broad categories: typical and atypical. Typical skull base osteomyelitis occurs secondary to uncontrolled infection of the temporal bone region, most often from necrotizing external otitis caused by Pseudomonas aeruginosa in a patient with diabetes. Atypical skull base osteomyelitis occurs in the absence of obvious temporal bone infection or external auditory canal infection. It may be secondary to advanced sinusitis or deep face infection or might occur in the absence of a known local source of infection. Atypical skull base osteomyelitis preferentially affects the central skull base and can be caused by bacterial or fungal infections. Clinically, typical skull base osteomyelitis presents with signs and symptoms of otitis externa or other temporal bone infection. Both typical and atypical forms can produce nonspecific symptoms including headache and fever, and progress to cranial neuropathies and meningitis. Early diagnosis can be difficult both clinically and radiologically, and the diagnosis is often delayed. Radiologic evaluation plays a critical role in the diagnosis of skull base osteomyelitis, with CT and MR imaging serving complementary roles. CT best demonstrates cortical and trabecular destruction of bone. MR imaging is best for determining the overall extent of disease and best demonstrates involvement of marrow space and extraosseous soft tissue. Nuclear medicine studies can also be contributory to diagnosis and follow-up. The goal of this article was to review the basic pathophysiology, clinical findings, and key radiologic features of skull base osteomyelitis.
\end{abstract}

ABBREVIATIONS: ASBO = atypical skull base osteomyelitis; EAC = external auditory canal; Ga-67 = gallium-67 citrate; lgG4 = immunoglobulin G4; Tc99m MDP = technetium TC99m methylene diphosphonate; NEO = necrotizing external otitis; SBO = skull base osteomyelitis; TSBO = typical skull base osteomyelitis

S kull base osteomyelitis (SBO) is a rare, potentially life-threatening infection that can present a diagnostic challenge clinically and radiologically. ${ }^{1-4}$ While reports differ in terminology, there are generally 2 categories of SBO: typical and atypical. Typical SBO (TSBO) is the most common and classically occurs in elderly patients with diabetes as a result of necrotizing external otitis (NEO) caused by Pseudomonas species. (Fig 1). ${ }^{1,3}$ Atypical SBO (ASBO), also called central SBO, predominantly involves the basisphenoid and basiocciput and occurs without preceding otologic infection (Fig 2). ${ }^{2,5}$ Recognition of SBO is increasing, and it is clear that radiologic evaluation plays a critical role in diagnosis and management. The goal of this article was to review the

Received May 8, 2020; accepted after revision August 21.

From the Section of Neuroradiology, Department of Radiology, University of Alabama at Birmingham, Birmingham, Alabama.

Please address correspondence to Aparna Singhal, MD, Department of Radiology, University of Alabama at Birmingham, 619 19th St South, Birmingham AL 35249; e-mail: asinghal@uabmc.edu

- Indicates open access to non-subscribers at www.ajnr.org

http://dx.doi.org/10.3174/ajnr.A7015 pathophysiology, clinical presentation, and detailed radiologic findings using multiple modalities including CT, MR imaging, and nuclear medicine.

\section{TSBO}

TSOB is considered a part of the NEO spectrum, and these terms are often used interchangeably. ${ }^{1,2,6}$ TSBO can also occur secondary to other otologic infections, including complicated otomastoiditis or petrous apicitis. ${ }^{4,8}$ It can also occur secondary to trauma or as a surgical complication..$^{7-10}$

Disease may begin as localized otitis externa (Fig 3). Progressive, deeper infection leads to NEO, in which microorganisms invade local cartilage and bone, spreading through natural gaps in the cartilaginous framework of the external auditory canal (EAC) (fissures of Santorini). ${ }^{1}$ Infection can extend from the EAC through the foramen of Huschke into the temporomandibular joint (Fig 4). Progression of NEO ultimately causes TSOB, with local osseous destruction and localized marrow infiltration. From the EAC, infection most often spreads anteromedially to the infratemporal and preclival soft tissues, petrous apex, and clivus (Fig 5). ${ }^{1}$ 
Patients with TSBO have otorrhea and severe otalgia, with pain often out of proportion to the physical findings. Local adenopathy may be present, but fever and leukocytosis are often absent. ${ }^{1}$ The erythrocyte sedimentation rate is generally increased and can be used to monitor treatment. Trismus may occur with involvement of the masticator space. Infiltration of the EAC or nasopharyngeal soft tissues may produce mass effect and suggests underlying malignancy. Eustachian tube obstruction can cause further fluid accumulation and phlegmon in the middle ear. Cranial neuropathies can occur and may indicate a poor prognosis. Facial nerve palsy is seen in $25 \%$ of patients as the disease spreads medially to involve the stylomastoid foramen. ${ }^{1,11}$ Extension to the petrous apex may result in Gradenigo syndrome (facial pain, cranial nerve VI palsy, and persistent otorrhea). ${ }^{12}$

Inferomedial spread of infection to the jugular foramen and carotid space can result in multiple lower cranial neuropathies (cranial nerves IX-XII). ${ }^{1,2}$ Involvement of the sympathetic plexus along the internal carotid artery can also produce Horner syndrome. Rare cases of Villaret syndrome (neuropathies of


FIG 1. Typical skull base osteomyelitis. A 55-year-old man with type 2 diabetes with low-grade fever, severe pain, and drainage from his left ear. Eight weeks before imaging, the patient had been diagnosed with left-sided otitis media and possible otitis externa. He had initially been treated with amoxicillin and ofloxacin drops, without improvement. Culture from left-ear drainage revealed methicillin-sensitive $S$ aureus. The patient was treated with IV vancomycin and piperacillin/tazobactam for 2 weeks and then 14 weeks of amoxicillin/clavulanate. All clinical and laboratory parameters initially resolved during 4 months. However, a gallium scan continued to indicate abnormal activity of the skull base at both 8 and 16 weeks. Despite the gallium scan, antibiotics were discontinued. Within 2 weeks, the patient had recurrent symptoms and severe neck pain. Additional imaging (not shown) indicated new cervicocranial septic arthritis, requiring an additional 3 months of IV antibiotics that led to a cure. A, Axial CT demonstrates opacification of left-mastoid air cells. There is subtle erosion along the petro-occipital fissure (arrow) and loss of cortical bone along the left lateral margin of the clivus (arrowhead). B, Axial unenhanced T1weighted MR image demonstrates abnormal signal in the marrow space of the basiocciput (arrow), ill-defined signal in the left carotid space, and masslike submucosal infiltration of the left nasopharynx (arrowhead). C, Axial T1-weighted fat-saturated contrast-enhanced image demonstrates abnormal enhancement involving the marrow space of the clivus (arrow) as well as heterogeneous enhancement of infiltrating soft tissue in the left nasopharynx (arrowhead). cranial nerves IX-XII plus Horner syndrome) have been reported when SBO affects the jugular foramen. ${ }^{13}$ Secondary thrombophlebitis of the jugular bulb and sigmoid sinus can also occur. ${ }^{11}$ The internal carotid artery can be affected anywhere from the neck to the cavernous sinus, producing infectious arteritis, thrombosis, pseudoaneurysm, and stroke.

Intracranial spread can result in meningitis, epidural abscess, and cavernous sinus thrombosis. If the cavernous sinus is affected, multiple upper cranial nerves can be involved. ${ }^{11}$ Cranial nerve involvement is typically unilateral but can be bilateral in advanced SBO crossing the midline.

Patients with diabetes are particularly prone to NEO/TSBO due to a combination of immune dysfunction and microvascular angiopathy. Pseudomonas aeruginosa is responsible for $98 \%$ of cases. ${ }^{14}$ The virulence of this Gram-negative bacterium is related to angioinvasion and small-
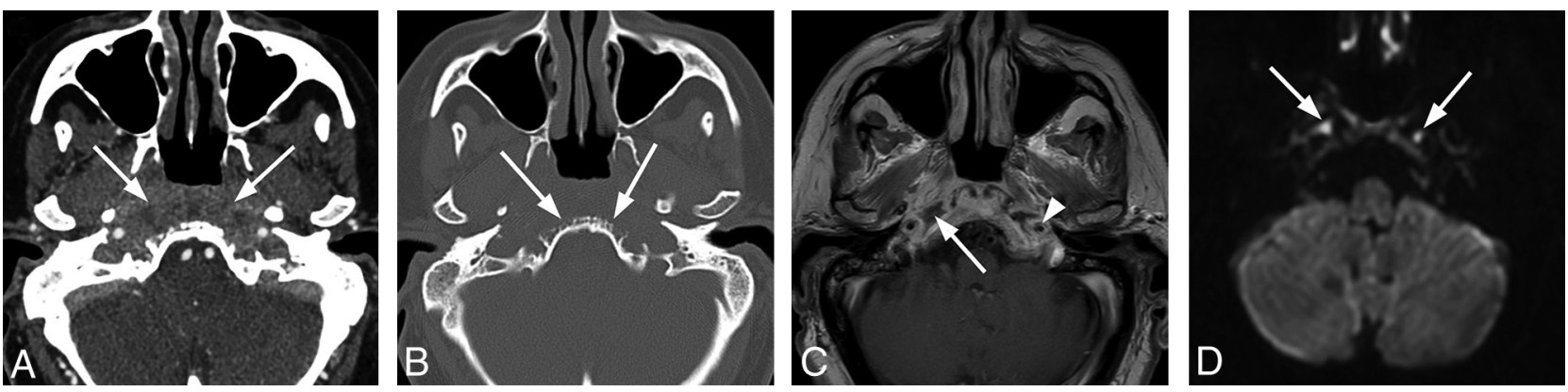

FIG 2. Atypical skull base osteomyelitis. A 72-year-old man with history of hypertension presented with a 9-week history of sinus congestion, rhinorrhea, and headache. The patient had been treated for severe sinusitis with several courses of oral antibiotics and steroid injections prescribed by his primary care physician as well as community ear, nose, and throat physicians. Just before admission, he developed left hearing loss and left-sided facial palsy. Swab culture of the nasopharynx revealed $P$ aeruginosa. The patient was treated with amoxicillin-pot clavulanate (Augmentin) and Ceftazidime (Ceftaz) with clinical resolution after several weeks. Follow-up MR imaging at 4 months confirmed improvement. $A$, Axial contrast-enhanced CT scan demonstrates patchy heterogeneous density in the preclival soft tissues extending to involve the carotid spaces bilaterally (arrows). The inflammatory tissues in the preclival soft tissues could mimic an infiltrative neoplasm of the nasopharynx. $B$, Axial bone CT at the same level shows irregular erosions of the ventral clivus (arrows). C, Axial T1-weighted contrast-enhanced MRI. There is nodular enhancement involving the clivus at this level with focal areas of necrosis and abscess just inferior to the foramina lacerum (arrow). Note circumferential enhancement of the petrous internal carotid arteries (arrowhead). D, Axial DWI depicts small foci of fluid near the foramen lacerum bilaterally as diffusion-restricted, consistent with focal abscesses (arrows). 

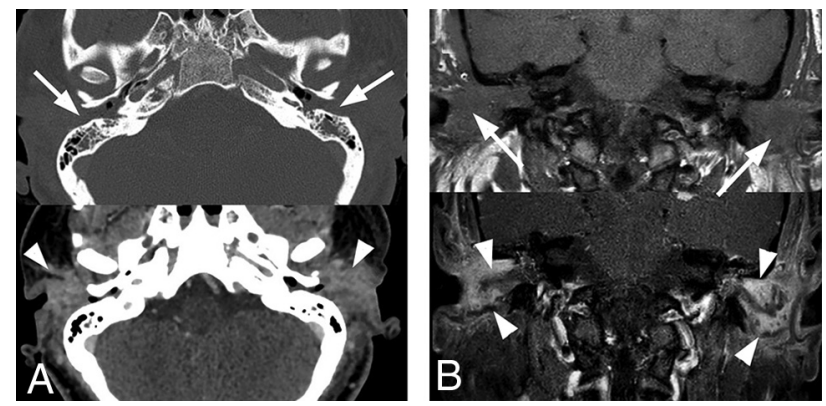

FIG 3. Bilateral external otitis. A 44-year-old woman presented with severe bilateral ear pain and drainage with conductive hearing loss. Clinically, there was marked inflammatory thickening of the EACs bilaterally. Pseudomonas species were cultured from the external auditory canals bilaterally. The patient was treated with IV vancomycin and piperacillin/tazobactam for 4 days followed by 2 weeks of oral ciprofloxacin. Symptoms resolved at 3 weeks without additional imaging work-up. A, Axial enhanced CT images are shown. Upper image with bone windows shows marked opacification of the EACs (arrows). The lower image with a soft-tissue filter shows marked inflammation of the EACs and periauricular tissues (arrowheads). B, The upper coronal $\mathrm{T} 1$ image shows marked opacification and thickening of the EACs (arrows) and associated opacification of the middle ear cavities. The lower image depicts postcontrast fat-saturated $\mathrm{T1}$ images. A discrete mass is not identified. However, there is marked enhancement along the walls of the EACs (arrowheads), compatible with otitis externa.
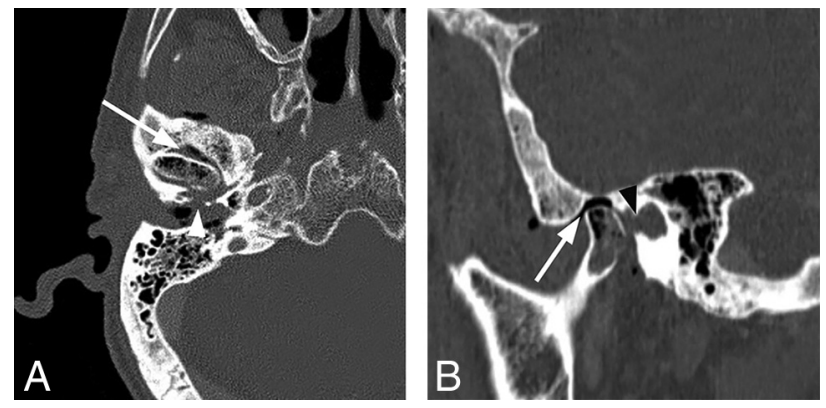

FIG 4. Necrotizing external otitis complicated by septic temporomandibular joint arthritis. An 88-year-old man with several months of right ear pain and drainage presented with progressive symptoms and right temporomandibular joint pain and trismus. In the prior weeks, he had been treated for NEO with oral and IV antibiotics at an outside facility. After initial evaluation, debridement and temporomandibular joint replacement were planned, but the patient was lost to follow-up. A, Axial contrast-enhanced CT with bone windows demonstrates abnormal opacification of the right EAC and inferior middle air cavity. There is erosion of the floor of the EAC, potentially involving the foramen of Huschke (arrowhead), and communication with the right temporomandibular joint. Air/gas density is identified within the temporomandibular joint (arrow) as well as within the mandibular condyle itself. $B$, Sagittal reformatted $C T$ scan again demonstrates abnormal air density within the right temporomandibular joint and mandibular condyle (arrow). Note a defect in the anterior inferior margin of the EAC (black arrowhead).

vessel thrombosis. ${ }^{1}$ Rarely, other bacteria including Staphylococcus species are reported. Otologic fungal infections, especially Aspergillus species, are increasingly reported in immunocompromised patients and can lead to fungal SBO.,15
TSBO has been traditionally associated with high morbidity and mortality despite intensive antibiotic therapy, with reported survival rates of around 50\%. ${ }^{2,16}$ Although prognosis has improved, recent studies still report mortality of up to $30 \%$, and it is still difficult to cure. ${ }^{17-19}$

\section{ASBO}

$\mathrm{ASBO}$, or central $\mathrm{SBO}$, for has a predilection for the clivus and occurs without precipitating otologic infection. It can be idiopathic or secondary to regional infections of the sinus, deep face, or oral cavity. ${ }^{4,8,10,16,19}$ The distinction between typical and atypical is not always clear because patients may have occult or partially treated infection before diagnosis of SBO. Furthermore, infection of the temporal bone can spread medially to the central skull base and vice versa, making the true origin uncertain in some cases. ${ }^{6,20}$

The clinical features of ASBO are nonspecific. Patients are generally middle-aged to elderly with underlying diabetes or other immunocompromised states (HIV, chronic steroid use, and so forth). ${ }^{2}$ Ridder et $\mathrm{al}^{18}$ reported that $70 \%$ of patients with ASBO had a predisposing factor affecting bone vascularization, including diabetes (45\%). Additional predisposing factors include previous radiation therapy, anemia, malnutrition, chronic cardiopulmonary disease, Paget disease, and osteoporosis. Rare patients have ASBO with no relevant pre-existing illness.

The most common symptoms of ASBO are headache and cranial neuropathies, with sinonasal symptoms reported in $25 \%{ }^{2}$ Fever is uncommon, found in $<20 \%{ }^{2,3,5}$ The erythrocyte sedimentation rate can be elevated, but leukocytosis is absent more often than not. ${ }^{3,5}$ ASBO can begin with persistent sinus or other local infections, with spread from pneumatized space or soft tissues to the osseous skull base. Involvement of preclival or nasopharyngeal soft tissues raises concern for nasopharyngeal neoplasm. Intracranial extension can lead to meningitis, multiple cranial neuropathies, and cavernous sinus thrombosis. ${ }^{2,5,16}$

Gram-positive bacteria, including Staphylococcus species, are more common than Pseudomonas species. ${ }^{2,3}$ ASBO can be caused by fungal organisms, especially mucoraceal family in ketoacidosis and Aspergillus species in patients with neutropenia (Fig 6). 3,15,21 Nontuberculous Mycobacteria species are being increasingly recognized in the immunocompromised population. Some cases are polymicrobial.

In ASBO, a 90.5\% survival rate has been reported with aggressive management at 18-month follow-up, though up to one-third of the patients experienced residual neurologic sequelae. ${ }^{2}$

A summary of the comparison of clinical characteristics of TSBO and ASBO is shown in the Table.

\section{Pathology}

Biopsy is often necessary in the clinical course to exclude neoplasm, examine features of non-neoplastic tissue, and obtain direct microbiologic specimens for Gram stain, culture, and antibiotic-sensitivity assessment. In cases of SBO, pathologic specimens show inflammatory changes that vary from edema to purulence, with varying degrees of tissue necrosis. Histology may not reveal microbes directly, and cultures are important for definitive diagnosis and specific treatment. ${ }^{5}$ However, there have been 

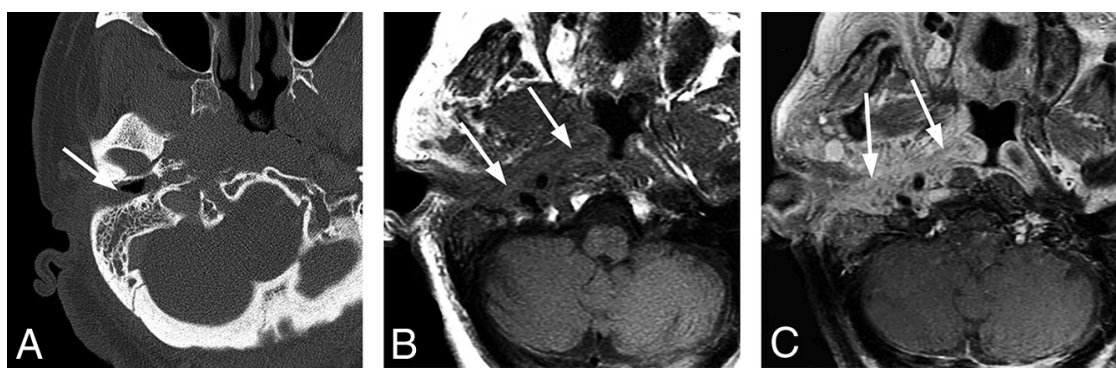

FIG 5. Typical SBO from NEO. An 82-year-old woman with insulin-dependent diabetes presented with a several-month history of pain and drainage from the right ear. The patient was recently treated as an outpatient with several rounds of antibiotics (including amoxicillin/clavulanate and ciprofloxacin) for otitis media and NEO, but symptoms had progressed. Following imaging and a diagnosis of SBO, the patient was treated with 4 weeks of IV vancomycin and piperacillin/tazobactam and additional 4 weeks of oral ciprofloxacin. The patient initially had clinical improvement, but a gallium scan at 8 weeks showed persistent uptake. The patient did not return for follow-up as instructed but returned 8 months after the original admission with progressive infection extending to the central skull base and left temporal bone, requiring an additional 6 weeks of IV antibiotic treatment. A, Axial CT scan through the right EAC demonstrates thickening and partial opacification of the external auditory canal (arrow). There is subtle erosion along the posterior wall of the external auditory canal and mild soft-tissue fullness of the right nasopharynx. B, Axial T7-weighted MR image demonstrates obliteration of normal fat planes in the submucosal and preclival tissues of the nasopharynx (arrows). This process extends posterolaterally to the tip of the mastoid on the right. C, Axial T1-weighted contrast-enhanced image demonstrates heterogeneous enhancement of abnormal tissue extending from the tip of the mastoid on the right to the nasopharynx (arrows) without discrete abscess formation.

emerging reports of culture-negative SBO, typically in patients with previous incomplete/partial treatment with topical or oral antibiotics. ${ }^{22,23}$ There is a wide range of reported culture-negative cases in the literature. ${ }^{24-26}$ In a systematic review by Mahdyoun et $\mathrm{al}^{26}$ culture-negative cases of NEO in the literature ranged from $0 \%$ to $36 \%$. Djalilian et al, ${ }^{22}$ in 2006 , reported 8 cases of SBO, all of which were culture-negative. Some authors have reported $100 \%$ positive cultures in their studies of patients with $\mathrm{SBO},{ }^{27}$ whereas some have reported up to $70 \%$ of patients with negative cultures, ultimately requiring empiric treatment. ${ }^{28}$ These data are limited by the absence of prospective studies and small sample sizes; a systematic analysis of these data to assess differences between typical and atypical SBO in this regard is beyond the scope of this article.

Imaging. Radiologic evaluation is critical for prompt diagnosis of SBO. The imaging approach depends on presenting symptoms. However, in general, a combination of complementary studies using high-resolution bone CT and gadolinium-enhanced MR imaging is often necessary. In challenging cases, molecular imaging studies can provide functional and metabolic information.

CT. Unenhanced CT is often first-line for suspected head and neck infections and is adequate for identifying opacification, mucosal thickening, and air-fluid levels in the temporal bones and sinuses. High-resolution submillimeter-section CT using a bone algorithm can be reformatted in multiple planes and is the study of choice for identifying cortical bone erosion or trabecular demineralization that accompanies osteomyelitis.

In suspected TSBO, it is critical to assess cortical bone loss, which can be subtle. In areas without prominent marrow and trabecular bone, this may be the only clue to SBO. Several key areas should be evaluated, including the bony EAC, mastoid tip, temporomandibular joint, petrous apex, petro-occipital fissure, foramen lacerum, jugular foramen, and clivus. In cases of ASBO, there is often evidence of invasive sinusitis with cortical erosions of the paranasal sinuses, particularly the sphenoid or ethmoid sinuses and possibly along the anterior clivus and foramina of the central skull base. ${ }^{5}$

Contrast-enhanced CT with softtissue windows can also be useful. In TSBO, the anteromedial spread of cellulitis and phlegmon in the infratemporal soft tissues manifests as poorly defined enhancement and soft-tissue infiltration. Occasionally, a frank abscess can be identified in the preclival soft tissues. Asymmetric soft-tissue fullness of the nasopharynx is common and can mimic an infiltrating neoplasm, especially nasopharyngeal carcinoma (Fig 7A). The soft-tissue invasion and neurovascular complications can occur before or without frank bone destruction, especially in early or aggressive diseases such as fungal SBO. CTA or CTV can be of additional benefit for evaluation of vascular complications, including cavernous sinus thrombosis or stroke.

MR Imaging. MR imaging of the skull base is complementary to $\mathrm{CT}$ and is superior for evaluating soft-tissue extent, marrow involvement, and intracranial complications related to $\mathrm{SBO}{ }^{3} \mathrm{~A}$ combination of MR images is necessary to fully evaluate the skull base and surrounding structures, including T1, T2, STIR, DWI, and T1-weighted fat-saturated contrast-enhanced images. Early TSBO may demonstrate abnormal signal and enhancement of the EAC, with marked edema and inflammation of the auricular soft tissues. With progression, there is anteromedial spread as described above.

On T1 images, the ill-defined soft-tissue process demonstrates hypo- or isointensity to muscle and causes obliteration of normal fat planes: retromandibular fat, parapharyngeal fat, and retropharyngeal fat in the preclival region. The infiltration consists of inflammation, edema, and phlegmon and produces T2 hyperintensity and heterogeneous enhancement on T1-weighted fat-saturated contrast-enhanced sequences. As noted, the soft-tissue abnormality in the nasopharynx may be the dominant feature and can be indistinguishable from an infiltrative neoplasm (Fig $7 B,-C)$.

When osteomyelitis affects the bone marrow, there is loss of normal fat signal in the marrow space, causing T1 hypointensity and STIR hyperintensity. The affected marrow demonstrates heterogeneous gadolinium enhancement. ${ }^{4,7,21}$ With advanced infection, bone marrow may become necrotic and evolve into an abscess, producing a region of peripherally enhancing tissue. 



FIG 6. Extensive atypical skull base osteomyelitis secondary to invasive fungal sinusitis (mucormycosis). A 68-year-old man with insulin-dependent diabetes mellitus and a history of chronic sinusitis and previous sinus operations presented with a 6-month history of severe headache, nausea and vomiting, and weight loss. Endoscopic debridement and biopsies were performed. While cultures were negative for fungus, pathologic evaluation of sinus material yielded microscopic evidence of invasive fungal sinusitis consistent with mucormycosis. The patient was treated with micafungin and amphotericin B. A protracted course was complicated by persistent symptoms, and the patient had additional sinonasal debridement at 4 months. Endoscopic culture at that time yielded $P$ aeruginos $a$ as a new or potentially coexistent organism, and IV ceftazidime was added to treatment. The patient was followed clinically and with CT/MR imaging until resolution. A, Axial unenhanced $\mathrm{CT}$ image through the skull base demonstrates diffuse osteolysis and fragmentation of the sphenoid bone, including the walls of the sphenoid sinus and greater wing (arrows). There is marked mucosal thickening and opacification of the visualized sinuses. B, Axial CT image through the central skull base shows diffuse bone demineralization of the body of the sphenoid bone (arrows). C, Axial Tl-weighted fat-saturated contrast-enhanced image shows abnormal enhancement in the greater wings of the sphenoid bone bilaterally (arrows) and confluent opacification of the sinuses. There is evidence of devitalization and necrosis in the upper clivus (arrowhead). D, Axial T1-weighted fat-saturated contrast-enhanced image shows marked infiltrative signal abnormality and enhancement in the greater wings of the sphenoid bone (arrows). There is marrow necrosis and devitalization of the body of the sphenoid bone centrally (arrowheads). E, Axial fused Tc99m MDP bone scan SPECT image shows marked radiotracer uptake in the greater wing of the sphenoid bone on the left and the anterior midline skull base, consistent with osteomyelitis. F, Axial fused TC99m MDP bone scan SPECT image shows multifocal areas of radiotracer uptake in the sphenoid bone bilaterally.

Mucormycosis infection, in particular, can produce a combination of abnormal enhancement and nonenhancing areas of devitalized soft tissue and bone. ${ }^{15,21}$

Early in TSBO, the volume of marrow space abnormality may be small and attention to detail and use of fat-suppression are necessary to identify subtle involvement of mastoid bone, petrous apex, or occipital bone. Progression of disease can lead to more diffuse involvement of the marrow space and can include the clivus. While most cases will present as unilateral abnormalities, disease can progress to bilateral skull base involvement.

With ASBO, the MR signal abnormalities of the affected soft tissues and the bone marrow will be similar to those in TSBO. The primary difference is that the epicenter of disease will be the sphenoid bone. Paranasal sinus opacification may be conspicuous. The primary marrow signal abnormality will be in the clivus but may also involve the lesser and greater wings of the sphenoid or petrous apices. The soft-tissue abnormalities will also involve the preclival soft tissues but may be more symmetric, producing diffuse fullness in the nasopharynx. Soft-tissue infiltration of the pterygopalatine fossa with obliteration of normal fat in these regions is typical of invasive sinusitis associated with SBO.

DWI, especially non-EPI DWI, may be of benefit in evaluating SBO. Diffusion restriction in nonenhancing fluid collections can help confirm an abscess. Additionally, ADC values may allow distinction between $\mathrm{SBO}$ and neoplasm, with bacterial SBO values shown to be higher than those in nasopharyngeal carcinoma or lymphoma. ${ }^{17}$ On postcontrast imaging, focal abscesses could show a peripheral rim of enhancement, whereas a neoplasm would generally demonstrate enhancement within the diffusion-restricting tissue. ${ }^{29}$

CT or MR imaging is not necessarily helpful in long-term monitoring of the disease because radiologic findings lag behind clinical improvement. ${ }^{1,11,30}$ Overall, improvement in soft-tissue findings is the best radiologic indicator of early improvement, ${ }^{11,31}$ but abnormalities of bone may persist for weeks to months despite a clinical response to treatment. ${ }^{1,3,5,31}$

Nuclear Medicine. Before the advent of CT and MR imaging, nuclear imaging served as a cornerstone for evaluation of $\mathrm{SBO}^{32}$ The various radionuclide studies provide functional and metabolic information that can help confirm and localize infection of the skull base and can be complementary to clinical findings and anatomic imaging to monitor treatment response.

Technetium Tc99m methylene diphosphonate (Tc99m MDP) can demonstrate increased osteoblastic bone activity that occurs in response to infection. A 3-phase Tc99m MDP bone scan is more sensitive than CT for early detection of SBO, with sensitivity approaching 100\%, including SPECT Tc99m MDP scans, which are reported to be more sensitive and a better prognosticator for patients with malignant external otitis. ${ }^{33}$ It typically shows abnormal increased tracer uptake in bone on all 3 phases (ie, immediate blood flow, blood pool [5-10 minutes], and delayed phase [34 hours]), whereas isolated soft-tissue infection will be differentiated by a normal delayed phase. If available, delayed-phase SPECT improves anatomic localization. A bone scan, however, lacks specificity for infection because it can demonstrate abnormal bone 


\begin{tabular}{|c|c|c|}
\hline & Typical SBO & Atypical SBO \\
\hline Age & Elderly & Middle-aged \\
\hline Predisposing factors & DM more common than immunocompromised & DM, immunocompromised \\
\hline Clinical features & $\begin{array}{l}\text { Otorrhea, otalgia (severe, with pain out of } \\
\text { proportion to the physical findings), hearing loss }\end{array}$ & $\begin{array}{l}\text { Headache, atypical facial pain, cranial } \\
\text { neuropathies, sinonasal symptoms in } 25 \%\end{array}$ \\
\hline Cranial nerve involvement & VII most common & $\mathrm{VI}, \mathrm{IX}$, and $\mathrm{X}$ more common than VII \\
\hline Pathogen & $\begin{array}{l}P \text { aeruginosa in most cases; fungal more common } \\
\text { in immunosuppressed patients without diabetes }\end{array}$ & $\begin{array}{l}S \text { aureus slightly more common than } \\
P \text { aeruginosa and fungal }\end{array}$ \\
\hline $\begin{array}{l}\text { Primary epicenter of } \\
\text { disease process }\end{array}$ & EAC, petrous apex, and clivus & $\begin{array}{l}\text { Central skull base, sphenoid bone, or clivus with } \\
\text { or without evidence of regional infection of } \\
\text { the sinuses, deep face, or oral cavity }\end{array}$ \\
\hline
\end{tabular}

Note:-DM indicates diabetes mellitus.
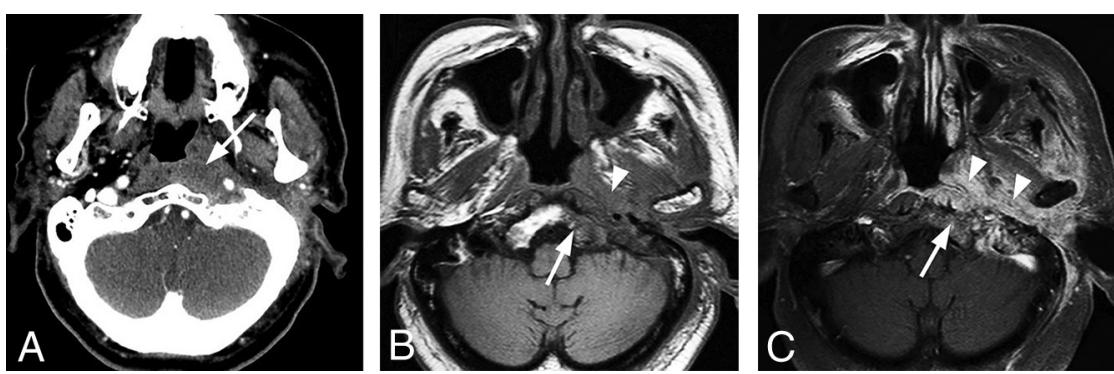

FIG 7. Typical SBO with atypical organisms. A 75-year-old man with diabetes presented with left-ear discharge, conductive hearing loss, and headache. Initial imaging suggested an infiltrative neoplasm of the nasopharynx, and multiple endoscopic biopsies of the nasopharynx were performed to exclude nasopharyngeal carcinoma. Ultimately, biopsies of the external auditory canal revealed an infectious organism, Aspergillus species. The patient was treated with amphotericin with gradual resolution of symptoms. A, Axial enhanced CT image through the level of the nasopharynx suggests an infiltrative soft-tissue abnormality involving the submucosa and preclival soft tissues of the nasopharynx (arrow). B, Axial Tl-weighted MR image through the nasopharynx shows poorly defined infiltrative soft tissue (arrowhead) in the submucosa of the nasopharynx on the left, extending to involve the left carotid space. There is also replacement of normal marrow on the left side of the basiocciput (arrow). C, Axial enhanced fat-saturated T1 image through the nasopharynx demonstrates abnormal enhancement on the left side of the occipital bone (arrow) as well as abnormal enhancing infiltrative tissue (arrowheads) in the preclival soft-tissue left carotid space and left retromandibular region. Courtesy of $\mathrm{Dr}$ Christine Glastonbury, Professor of Clinical Radiology, Otolaryngology Head and Neck Surgery, and Radiation Oncology, University of California, San Francisco, California.
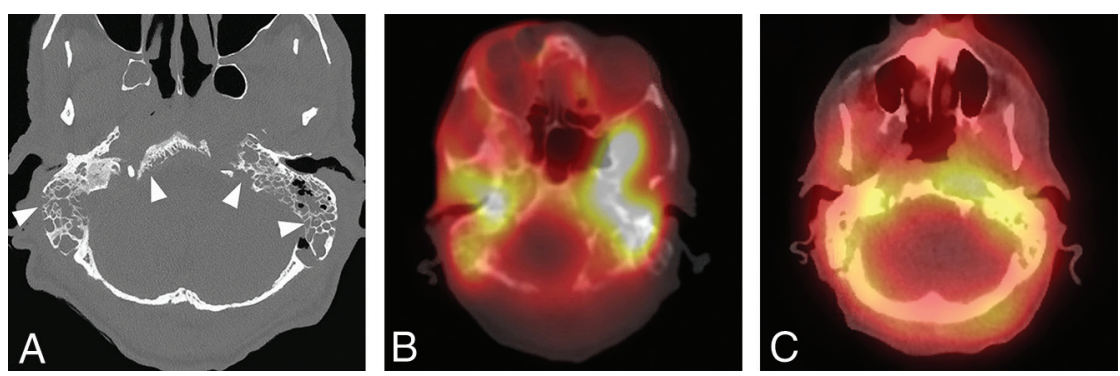

FIG 8. Extensive skull base osteomyelitis. A 70-year-old man with poorly controlled diabetes presented with symptoms of otomastoiditis with associated hearing loss, left facial nerve paralysis, and dysphagia. A culture of the left external auditory canal revealed Pseudomonas infection. The patient received aggressive treatment for several months, including IV vancomycin and piperacillin/tazobactam, but he ultimately died from meningitis and aspiration pneumonia. A, Axial CT demonstrates multiple focal areas of cortical dehiscence involving the temporal bones bilaterally as well as the clivus (arrowheads). The generalized erosion is compatible with diffuse osteomyelitis of the skull base. B, Axial fused Tc99m MDP bone scan SPECT image demonstrates significant accumulation of radiotracer in the skull base bilaterally, preferentially affecting the temporal bones. C, An axial fused gallium scan SPECT image shows bilateral accumulation of radiotracer in the temporal bones, with some extraosseous accumulation in the left preclival region. activity in malignancies, trauma, recent surgery, or noninfectious inflammation. Furthermore, in the setting of osteomyelitis, a bone scan can remain abnormal even after satisfactory treatment due to bone healing and remodeling (Fig 8). ${ }^{1,2,34,35}$

A gallium-67 citrate (Ga-67) scan targets acute-phase reactants like lactoferrin and bacterial siderophores and can bind to white blood cells engaged in the immune response to infection. This feature provides high specificity for infection and is complementary to the bone scan (Fig 9). A normal Ga-67 scan, even with an abnormal bone scan, reliably excludes SBO, and increased uptake on a Ga-67 scan confirms infection. A Ga-67 scan plays an important role in monitoring of treatment response, converting to normal findings after successful treatment; persistent increased uptake suggests residual infection. The scan can be repeated to monitor antibiotic response until findings become normal. ${ }^{6,34}$ This repetition can be reassuring for the consulting physician and the patient, especially in complicated cases in which the diagnosis was delayed or in doubt. The major limitation of a Ga-67 scan is the long scan time requiring delayed images up to 48-72 hours.

A technetium-labeled white blood cell scan is less commonly used but like Ga-67, it has a high specificity for SBO in the initial diagnosis. A tagged white blood cell study can be used for confirming healing at the end of antibiotic therapy. ${ }^{1,11,36}$

Overall, the literature has variable data regarding the overall diagnostic value of nuclear medicine 

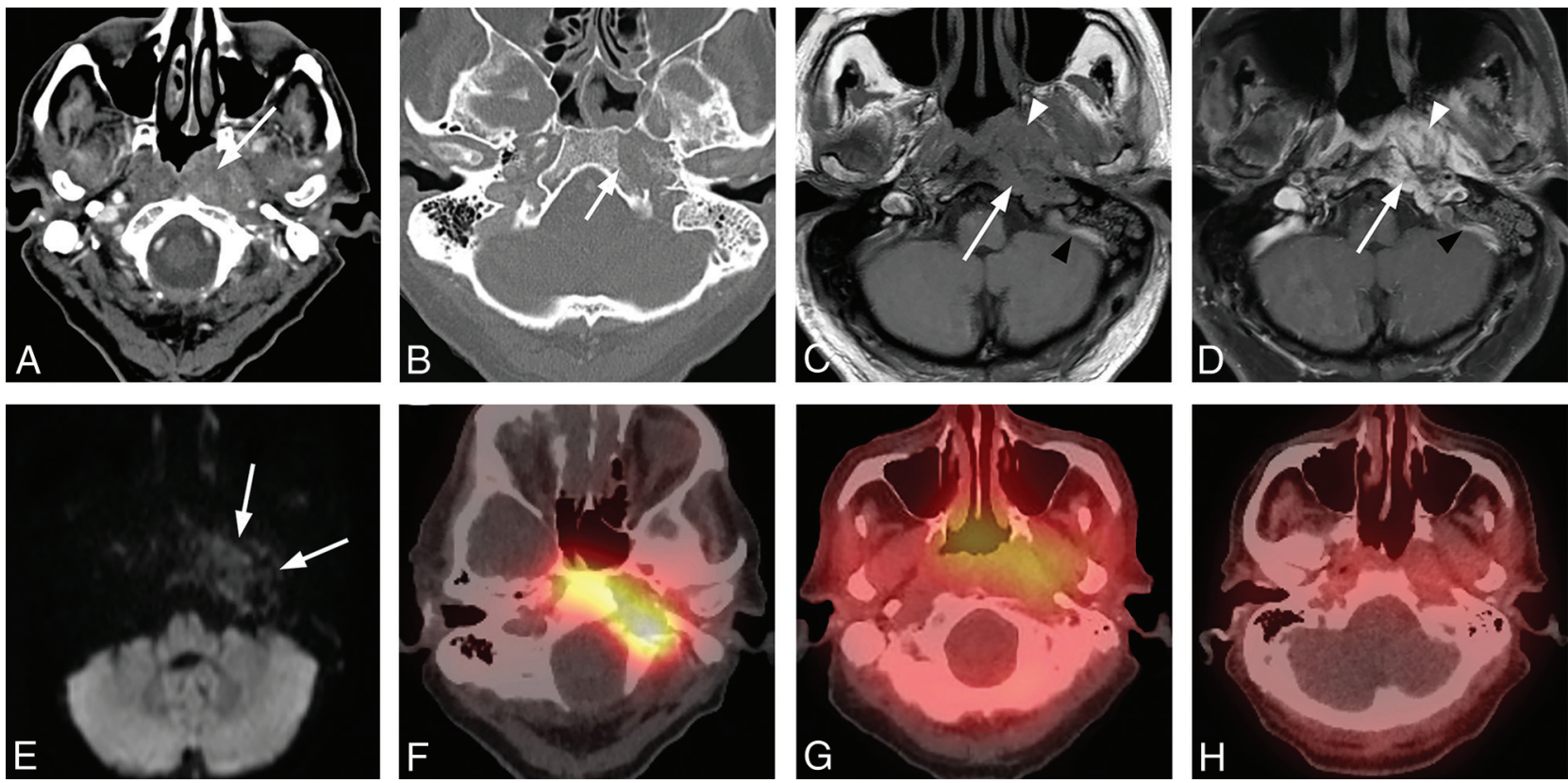

FIG 9. Typical skull base osteomyelitis. A 63-year-old man with diabetes presented to an outside hospital with a several-month history of left ear pain. He was initially diagnosed with otitis media and treated with several rounds of antibiotics. A recent evaluation at an outside hospital suggested the presence of a nasopharyngeal mass with skull base invasion. Two separate biopsies of the nasopharynx failed to demonstrate nasopharyngeal carcinoma. While a discrete organism was not cultured, the patient was presumptively treated with levofloxacin and clindamycin with gradual improvement and resolution of all symptoms. He was monitored using serial CT, MR imaging, and gallium scans. A, Axial postcontrast CT of the soft tissue of the neck at the level of the nasopharynx demonstrates a heterogeneously enhancing soft-tissue lesion involving the submucosal region of the left nasopharynx (arrow). The process extends laterally to the left carotid space, and there is occlusion or thrombosis of the left internal jugular vein. $B$, Axial postcontrast CT scan through the skull base with bone windows demonstrates focal cortical erosion along the margins of the foramen lacerum (arrow), consistent with osteomyelitis. C, Axial T--weighted MR image through the skull base shows an infiltrative process involving the left side of the clivus (arrow) and adjacent preclival tissues of the left nasopharynx. The lesion appears masslike on the left (arrowhead). The infiltrative process extends posterolaterally on the left to the jugular foramen. Partial thrombosis of the jugular bulb and sigmoid sinus is identified (black arrowhead). D, Axial enhanced T-weighted MR image through the skull base shows an enhancing infiltrative process involving the left side of the clivus (arrow) and adjacent preclival tissues of the left nasopharynx (arrowhead). The process extends posterolaterally on the left to the jugular foramen. Partial thrombosis of the jugular bulb and sigmoid sinus is identified (black arrowhead). E, Axial DWI shows no diffusion restriction in the nasopharyngeal soft tissue, favoring a non-neoplastic process over lymphoma or nasopharyngeal carcinoma. F, Axial fused Tc99m MDP bone scan SPECT image shows localized accumulation of radiotracer in the left skull base, compatible with osteomyelitis. G, An axial fused gallium scan SPECT image at the level of the nasopharynx shows mild uptake in the soft tissues of the nasopharynx. $H, A$ follow-up fused gallium scan SPECT image at the level of the nasopharynx demonstrates resolution of previously seen uptake in the nasopharyngeal soft tissues, favoring a treatment response.

studies. ${ }^{37}$ A recent review of malignant otitis externa literature revealed pooled sensitivities for technetium-99 and gallium-67 of $85.1 \%$ and $71.2 \%$, respectively, with poor specificity; however, the data were deemed insufficient for a meta-analysis. The authors, therefore, advised against the routine use of these studies in SBO management in patients with a known diagnosis on conventional imaging. However, these examinations were considered to be reasonably sensitive tests in patients with an unclear diagnosis despite an otomicroscopic examination or other imaging studies. ${ }^{37}$ The authors also concluded that there were insufficient data to determine the usefulness of these modalities during followup and that larger prospective studies would be necessary.

$\left[{ }^{18} \mathrm{~F}\right]$ FDG- PET detects increased glucose metabolism. FDG is nonspecific and accumulates at sites of high glucose demand, including active infection, but also in postoperative, inflammatory, or neoplastic tissue. The advantages of FDG-PET/CT over other nuclear studies are wider clinical availability, shorter imaging time, and higher spatial resolution. It can be complementary to determine the extent of infection in confirmed cases of $\mathrm{SBO}$ and for evaluation of treatment response. In a recent study comparing the diagnostic performance of $\left[{ }^{18} \mathrm{~F}\right]$ FDG-PET/CT with MR imaging, both modalities had comparable sensitivities (87.5 versus $81.25 \%$, respectively), but PET-CT had better specificity ( $71.0 \%$ versus $28.5 \%$, respectively) in identifying infection. The sensitivity, specificity, positive predictive value, negative predictive value, and accuracy of FDG-PET/CT in detecting SBO were 96.7\%, 93.3\%, $98.3 \%, 87.5 \%$, and $96.1 \%$, respectively. ${ }^{38}$ With wider availability of hybrid PET-MR imaging scanners with superior soft-tissue detail and metabolic information in a single imaging session, PET-MR can be used to follow patients with SBO. ${ }^{6,38,39}$ PET-MR imaging with gadolinium in combination with high-resolution $\mathrm{CT}$ is an excellent approach for suspected new SBO, and a combination of FDG-PET with either MR imaging or CT may provide sufficient follow-up (Fig 10). ${ }^{6}$

Differential Considerations. The primary diagnostic dilemma for SBO arises from neoplastic processes because they can also infiltrate the skull base and the adjacent soft tissues. Carcinoma of the EAC can have similar clinical and radiologic features to 



FIG 10. Progressive left-sided skull base osteomyelitis. A 65-year-old man with multiple comorbidities, including poorly controlled diabetes, presented with a relatively long-standing history of chronic sinusitis and bilateral otitis media. He was recently treated for otitis media of the left ear and presented with new headache and left-sided hearing loss. Culture from the left EAC was negative for a causative organism, and cultures from the sphenoid sinus demonstrated methicillin-resistant $S$ aureus. The patient was treated with 4 weeks of IV vancomycin and piperacillin/tazobactam. The patient improved, and a gallium scan performed during follow-up showed significant improvement and only mild residual uptake in the skull base. Antibiotics were discontinued. The patient returned 4 weeks later with sepsis, and blood cultures were positive for Klebsiella species. In the next 3 months, the patient had a return of headaches and new right-sided symptoms. Imaging evaluation demonstrated progressive infection of the right skull base. Re-institution of IV antibiotics led to gradual resolution of clinical and imaging findings. A, Axial T-weighted MRI through the skull base shows an infiltrative soft-tissue abnormality (arrows) involving the central skull base with abnormal marrow signal on the left side of the occipital bone (arrowhead). B, Axial enhanced Tweighted MR image shows heterogeneous enhancement in the corresponding areas of the left petro-occipital fissure (arrow). C, Axial bone scan fused with CT shows radiotracer accumulation in the left central skull base, including the sphenoid bone and left petrous apex. $D$, Following initiation of empiric IV antibiotic treatment, the patient had improvement of symptoms on the left, but 4 months later, he developed severe headache, fever, and right-sided facial pain. Axial T-weighted image shows an infiltrative process (arrows) of the central skull base that now extends to the right. $E$, Axial enhanced fat-saturated T-weighted image demonstrates improved enhancement of the left central skull base and the adjacent soft tissues but interval worsening of enhancement of the right skull base (arrowhead) and soft tissues of the nasopharynx (arrow). F, Follow-up axial fused bone scan SPECT image demonstrates radiologic worsening with marked radiotracer uptake in the central skull base in the right petro-occipital region.

those of TSBO and, therefore, needs to be excluded. Other neoplasms to consider are metastatic disease, nasopharyngeal carcinoma, sinonasal neoplasm, and lymphoma. ${ }^{3}$

Neoplasms that affect the skull base may arise locally from various sources that are amenable to clinical evaluation. For example, a primary skin lesion such as EAC squamous cell carcinoma or a primary mucosal lesion such as nasopharyngeal carcinoma may have a dominant, clinically obvious primary soft-tissue mass that heavily influences image interpretation and preliminary management decisions. Other neoplasms of the skull base such as lymphoma, myeloma, or metastatic disease may be accompanied by pertinent clinical history and other extracranial findings to indicate underlying systemic metastatic or multifocal disease and would typically present with more bulky lesions than ill-defined skull base pathologies.

Most primary forms of local invasive neoplasms that affect the skull base have a dominant soft-tissue mass or nodule that secondarily invades the bone. While the osseous skull base may have significant derangement due to invasion and destruction, the primary lesion presents on cross-sectional imaging as nodular or masslike enhancement in the extraosseous soft tissues. As opposed to SBO, malignant neoplasms typically displace or replace normal anatomy without preservation of tissue planes. Enhancement and fullness without destruction of fascial planes may support a diagnosis of SBO over tumor. ${ }^{29,40}$ Additionally, at presentation, MR imaging has been reported to show greater disease involvement compared with CT in central SBO, whereas malignancy generally shows equal involvement; and combining the $\mathrm{CT}$ and MR imaging information can help differentiate between central SBO and malignancy. ${ }^{40}$ With high-resolution imaging, especially T1-weighted contrast-enhanced fat-saturated MR imaging, the margins of the tumor can be reasonably mapped and measured. If a lesion involving the skull base has a dominant, solidly enhancing soft-tissue component that correlates with a clinically obvious mass involving the skin or mucosa, neoplasm is strongly favored over infection. In these cases, biopsy of the apparent lesion is indicated as initial management. In situations in which a primary lesion is not clinically apparent, imaging can help direct a potential biopsy of suspected viable tumor by identifying localized extraosseous soft-tissue enhancement. ${ }^{29}$

The dilemma is made more difficult in the setting of a small or occult primary tumor, deep subcutaneous or submucosal invasion, or tumor with significant ulceration or necrosis. In these cases, a dominant soft-tissue nodule or mass may be lacking both clinically and radiologically. This situation occasionally occurs in the setting of nasopharyngeal carcinoma or adenoid cystic carcinoma that affects the central skull base. In such circumstances, the radiologic picture may be dominated by osseous demineralization on CT and abnormal marrow space signal and enhancement on MR imaging. In these cases, infiltrating tumor can be difficult to distinguish from SBO. In addition, focal soft-tissue infection associated with SBO can produce phlegmon, occasionally taking on masslike qualities of soft-tissue fullness, mass effect, and enhancement.

The decision to biopsy tissue is based on multidisciplinary consultation. Surface mucosal or submucosal disease can be biopsied through endoscopic approaches by ear, nose and throat surgeons, whereas more deep-seated disease may require image- 
guided biopsies. Radiologic findings can provide specific clues in cases in which there have been repeat biopsies negative for malignancy. Alternate biopsy targets can be suggested on the basis of the imaging appearance to plan surgical approaches to the pterygopalatine fossa or orbital apex for biopsy and tissue analysis. ${ }^{23}$ Often during the course of the disease, these cases will need a multidisciplinary approach, with consultations among the referring clinician, surgeons, infectious disease specialists, and radiologists at different steps. ${ }^{23}$

Neoplasms that are intrinsic to the skull base such as chordoma or chondrosarcoma can be considered invasive or infiltrative, but they tend to be slow-growing, focally expansile, and relatively well-circumscribed. Tumor grows beyond the margins of the bone into adjacent soft tissues, but there is not typically an inflammatory response.

Rare differential considerations would include non-neoplastic diseases, including granulomatosis with polyangiitis and other granulomatous diseases (eg, tuberculosis, sarcoidosis). ${ }^{3}$ Idiopathic skull base inflammation (inflammatory pseudotumor), an idiopathic noninfectious inflammatory condition, may primarily involve the skull base or extend from the orbit and can appear identical to SBO. ${ }^{41}$ Immunoglobulin G4 (IgG4)-related disease can affect almost any organ, most commonly the submandibular, lacrimal, or parotid glands, but it can also involve the skull base. IgG4related disease typically shows increased IgG-4-positive plasma cells on tissue sampling, and elevated serum IgG4 concentrations are also seen. ${ }^{42}$ An elevated IgG4/IgG ratio of $>0.4$ was detected in $40 \%$ of cases in a study of inflammatory pseudotumor and helped to distinguish them from SBO in some instances because none of the SBO cases had a ratio of $>0.4 .{ }^{43}$ Ultimately, radiologic findings alone are insufficient to differentiate these inflammatory entities from SBO and malignancy. These entities often are suspected in the absence of a mass or signs of infection, but endoscopic biopsy/tissue sampling will be needed for diagnosis. ${ }^{23,43}$

Primary bone conditions of the skull base, including fibrous dysplasia and Paget disease, can be in the differential for SBO on MR imaging; however, CT would show their typical appearances with bony expansion and no associated soft-tissue abnormality. Ground glass opacification with variable lytic foci would be seen in fibrous dysplasia and osseous expansion with a lytic lesion (osteoporosis circumscripta) or mixed lytic-sclerotic foci having a cotton wool appearance as seen in Paget disease. ${ }^{3}$

Management/Treatment. TSBO often has a classic presentation and is not difficult to diagnose, whereas ASBO is often a diagnostic dilemma due to the nonspecific initial presentation. For any infiltrative/destructive process of the central skull base, neoplastic processes including nasopharyngeal carcinoma, lymphoma, or leukemia need to be ruled out first with other aforementioned inflammatory or noninflammatory conditions also considered. Skull base or nasopharyngeal biopsies need to be performed in a timely manner to rule out these differential possibilities as well as to obtain tissue samples due to the potential for rapid progression of SBO. ${ }^{11,18}$ Tissue samples should undergo microbial analysis with culture and flow cytometry for lymphoma in addition to pathologic analysis, especially if the clinical suspicion is high and no obvious soft-tissue lesions are seen. ${ }^{1,5}$ Pathogen-specific antibiotic therapy including IV antibiotics followed by long-term oral antibiotics would be the mainstay of treatment, currently recommended for 6-20 weeks, ${ }^{2,18,44}$ with wide variations in the duration of treatment observed in a survey-based study of otolaryngologists in the United Kingdom. ${ }^{45}$ Initially however, broadspectrum antimicrobials, including coverage for $P$ aeruginosa and methicillin-resistant Staphylococcus aureus particularly for nonotologic causes, are recommended to cover the possibility of polymicrobial infection in ASBO before culture and sensitivity information is available. ${ }^{18}$ Antipseudomonal antibiotics, such as carbapenems and third-generation cephalosporins, with ciprofloxacin in the long term, are considered an alternative to single initial therapy with ciprofloxacin in view of growing ciprofloxacin resistance in the intensive care setting, including in culture-negative cases, in which the antibiotic choice can be difficult. ${ }^{1,18,24}$ Surgical debridement of necrotic bone and soft tissue, especially for fungal disease, may be required in advanced cases with drainage of involved air cells or sinuses and of abscesses to also help improve antimicrobial penetration. However, an early and aggressive surgical approach has also been found to be beneficial and is recommended by some authors, especially in patients with prolonged ear infections and at the first signs of cranial neuropathy. ${ }^{18}$ Hyperbaric oxygen therapy has also been suggested as an ancillary treatment but has not shown an impact on survival. ${ }^{1,2}$

\section{Summary}

Diagnosis of SBO, clinically and radiologically, requires a high index of suspicion, and a delay in diagnosis is common. It should be considered in the differential consideration for any infiltrative skull base process, particularly if biopsies are negative for malignancy. Thin section, high-resolution bone CT of the skull base would be necessary to identify early cortical erosion followed by multiplanar pre- and postcontrast MR imaging to identify marrow space involvement. Nuclear medicine imaging studies can play an important role in difficult-to-diagnose cases and in follow-up. Long-term antibiotics with surgical debridement in advanced cases are the mainstay of management.

Disclosures: Philip R. Chapman—UNRELATED: Employment: University of Alabama Birmingham, Comments: I am an Associate Professor at University of Alabama; Payment for Lectures Including Service on Speakers Bureaus: Los Angeles Radiological Society, Comments: I received an honorarium for a total of 5 lectures at a recent annual meeting in Los Angeles, California, January 2020; Royalties: Elsevier, Comments: royalties for textbooks: 1) Chapman PR, Harnsberger HR, Vattoth S. Imaging Anatomy: Head \& Neck. 1st ed. Elsevier, 2018 (September): ISBN: 978-0323568722; Shaaban AM, ed, Diagnostic Imaging, Oncology, 2nd ed, November 2019, ISBN: 9780323661126. Gagandeep ChoudharyUNRELATED: Employment: University of Alabama at Birmingham.

\section{REFERENCES}

1. Carfrae MJ, Kesser BW. Malignant otitis externa. Otolaryngol Clin North Am 2008;41:537-49 CrossRef Medline

2. Johnson AK, Batra PS. Central skull base osteomyelitis: an emerging clinical entity. Laryngoscope 2014;124:1083-87 CrossRef Medline

3. Chang PC, Fischbein NJ, Holliday RA. Central skull base osteomyelitis in patients without otitis externa: imaging findings. AJNR Am J Neuroradiol 2003;24:1310-16 Medline

4. Borges A. Imaging of the central skull base. Neuroimaging Clin N Am 2009;19:669-96 CrossRef Medline 
5. Clark MP, Pretorius PM, Byren I, et al. Central or atypical skull base osteomyelitis: diagnosis and treatment. Skull Base 2009;19:247-54 CrossRef Medline

6. van Kroonenburgh A, van der Meer WL, Bothof RJ, et al. Advanced imaging techniques in skull base osteomyelitis due to malignant otitis externa. Curr Radiol Rep 2018;6:3 CrossRef Medline

7. Bag AK, Chapman PR. Neuroimaging: intrinsic lesions of the central skull base region. Semin Ultrasound CT MR 2013;34:412-35 CrossRef Medline

8. Prasad SC, Prasad KC, Kumar A, et al. Osteomyelitis of the temporal bone: terminology, diagnosis, and management. J Neurol Surg $B$ Skull Base 2014;75:324-31 CrossRef Medline

9. Chen JC, Yeh CF, Shiao AS, et al. Temporal bone osteomyelitis: the relationship with malignant otitis externa, the diagnostic dilemma, and changing trends. ScientificWorldJournal 2014;2014:591714 CrossRef Medline

10. Ducic Y. Skull base osteomyelitis. South Med J 2006;99:1051 CrossRef Medline

11. Adams A, Offiah C. Central skull base osteomyelitis as a complication of necrotizing otitis externa: imaging findings, complications, and challenges of diagnosis. Clin Radiol 2012;67:e7-16 CrossRef Medline

12. Holder CD, Gurucharri M, Bartels LJ, et al. Malignant external otitis with optic neuritis. Laryngoscope 1986;96:1021-23 Medline

13. Huang KL, Lu CS. Skull base osteomyelitis presenting as Villaret's syndrome. Acta Neurol Taiwan 2006;15:255-58 Medline

14. Rubin Grandis J, Branstetter BF, Yu VL. The changing face of malignant (necrotising) external otitis: clinical, radiological, and anatomic correlations. Lancet Infect Dis 2004;4:34-39 CrossRef Medline

15. Chan LL, Singh S, Jones D, et al. Imaging of mucormycosis skull base osteomyelitis. AJNR Am J Neuroradiol 2000;21:828-31 Medline

16. Chandler JR, Grobman L, Quencer R, et al. Osteomyelitis of the base of the skull. Laryngoscope 1986;96:245-51 CrossRef Medline

17. Ozgen B, Oguz KK, Cila A. Diffusion MR imaging features of skull base osteomyelitis compared with skull base malignancy. AJNR Am J Neuroradiol 2011;32:179-84 CrossRef Medline

18. Ridder GJ, Breunig C, Kaminsky J, et al. Central skull base osteomyelitis: new insights and implications for diagnosis and treatment. Eur Arch Otorhinolaryngol 2015;272:1269-76 CrossRef Medline

19. Singh A, Al Khabori M, Hyder MJ. Skull base osteomyelitis: diagnostic and therapeutic challenges in atypical presentation. Otolaryngol Head Neck Surg 2005;133:121-25 CrossRef Medline

20. Kwon $\mathrm{BJ}$, Han MH, Oh SH, et al. MRI findings and spreading patterns of necrotizing external otitis: is a poor outcome predictable? Clin Radiol 2006;61:495-504 CrossRef Medline

21. Alleyne CH, Jr, Vishteh AG, Spetzler RF, et al. Long-term survival of a patient with invasive cranial base rhinocerebral mucormycosis treated with combined endovascular, surgical, and medical therapies: case report. Neurosurgery 1999;45:1461-63; discussion 1463-64 CrossRef Medline

22. Djalilian HR, Shamloo B, Thakkar KH, et al. Treatment of culturenegative skull base osteomyelitis. Otol Neurotol 2006;27:250-55 CrossRef Medline

23. See A, Tan TY, Gan EC. Atypical culture-negative skull base osteomyelitis masquerading as advanced nasopharyngeal carcinoma. Am J Otolaryngol 2016;37:236-39 CrossRef Medline

24. Loh S, Loh WS. Malignant otitis externa: an Asian perspective on treatment outcomes and prognostic factors. Otolaryngol Head Neck Surg 2013;148:991-96 CrossRef Medline

25. Spielmann PM, Yu R, Neeff M. Skull base osteomyelitis: current microbiology and management. J Laryngol Otol 2013;127(Suppl 1):S812 CrossRef Medline
26. Mahdyoun P, Pulcini C, Gahide I, et al. Necrotizing otitis externa: a systematic review. Otol Neurotol 2013;34:620-29 CrossRef Medline

27. Le Clerc N, Verillaud B, Duet M, et al. Skull base osteomyelitis: incidence of resistance, morbidity, and treatment strategy. Laryngoscope 2014;124:2013-16 CrossRef Medline

28. Sokołowski J, Lachowska M, Karchier E, et al. Skull base osteomyelitis: factors implicating clinical outcome. Acta Neurol Belg 2019;119: 431-37 CrossRef Medline

29. Jain N, Jasper A, Vanjare HA, et al. The role of imaging in skull base osteomyelitis: reviewed. Clin Imaging 2020;67:62-67 CrossRef Medline

30. Rothholtz VS, Lee AD, Shamloo B, et al. Skull base osteomyelitis: the effect of comorbid disease on hospitalization. Laryngoscope 2008;118:1917-24 CrossRef Medline

31. Rubin J, Curtin HD, Yu VL, et al. Malignant external otitis: utility of CT in diagnosis and follow-up. Radiology 1990;174:391-94 CrossRef Medline

32. Mendelson DS, Som PM, Mendelson MH, et al. Malignant external otitis: the role of computed tomography and radionuclides in evaluation. Radiology 1983;149:745-49 CrossRef Medline

33. Balakrishnan R, Dalakoti P, Nayak DR, et al. Efficacy of HRCT imaging vs SPECT/CT scans in the staging of malignant external otitis. Otolaryngol Head Neck Surg 2019;161:336-42 CrossRef Medline

34. Chakraborty D, Bhattacharya A, Gupta AK, et al. Skull base osteomyelitis in otitis externa: the utility of triphasic and single photon emission computed tomography/computed tomography bone scintigraphy. Indian J Nucl Med 2013;28:65-69 CrossRef Medline

35. Strashun AM, Nejatheim M, Goldsmith SJ. Malignant external otitis: early scintigraphic detection. Radiology 1984;150:541-45 CrossRef Medline

36. Rozenblum-Beddok L, Verillaud B, Paycha F, et al. (99m)TcHMPAO-leukocyte scintigraphy for diagnosis and therapy monitoring of skull base osteomyelitis. Laryngoscope Investig Otolaryngol 2018;3:218-24 CrossRef Medline

37. Moss WJ, Finegersh A, Narayanan A, et al. Meta-analysis does not support routine traditional nuclear medicine studies for malignant otitis. Laryngoscope 2020;130:1812-16 CrossRef Medline

38. Kulkarni SC, Padma S, Shanmuga Sundaram P. In the evaluation of patients with skull base osteomyelitis, does 18F-FDG PET CT have a role? Nucl Med Commun 2020;41:550-59 CrossRef Medline

39. Louarn N, Alias Q, Aupin L, et al. A rare presentation of skull-base osteomyelitis with neurovascular sheath extension following external otitis resolved by PET/MRI. Eur J Nucl Med Mol Imaging 2018;45:2025 CrossRef Medline

40. Lesser FD, Derbyshire SG, Lewis-Jones H. Can computed tomography and magnetic resonance imaging differentiate between malignant pathology and osteomyelitis in the central skull base? $J$ Laryngol Otol 2015;129:852-59 CrossRef Medline

41. Lee EJ, Jung SL, Kim BS, et al. MR imaging of orbital inflammatory pseudotumors with extraorbital extension. Korean J Radiol 2005; 6:82-88 CrossRef Medline

42. Yim CD, An HJ, Ahn SK, et al. IgG4-related disease presenting as otogenic skull base osteomyelitis. Auris Nasus Larynx 2020 Feb 25. [Epub ahead of print] CrossRef Medline

43. Ryu G, Cho HJ, Lee KE, et al. Clinical significance of IgG4 in sinonasal and skull base inflammatory pseudotumor. Eur Arch Otorhinolaryngol 2019;276:2465-73 CrossRef Medline

44. Hsiao YC, Lee JC, Kang BH, et al. Idiopathic osteomyelitis at the base of the skull. South Med J 2006;99:1121-23 CrossRef Medline

45. Chawdhary G, Pankhania M, Douglas S, et al. Current management of necrotising otitis externa in the UK: survey of 221 UK otolaryngologists. Acta Otolaryngol 2017;137:818-22 CrossRef Medline 